\title{
Molecular mechanisms of HIV latency
}

\author{
Daniele C. Cary, Koh Fujinaga, and B. Matija Peterlin \\ Departments of Medicine, Microbiology and Immunology, UCSF, San Francisco, California, USA.
}

\begin{abstract}
HIV seeds reservoirs of latent proviruses in the earliest phases of infection. These reservoirs are found in many sites, including circulating cells, the lymphoid system, the brain, and other tissues. The "shock and kill" strategy, where HIV transcription is reactivated so that antiretroviral therapy and the immune system clear the infection, has been proposed as one approach to curing AIDS. In addition to many defective viruses, resting hematopoietic cells harbor transcriptionally latent HIV. Understanding basic mechanisms of HIV gene expression provides a road map for this strategy, allowing for manipulation of critical cellular and viral transcription factors in such a way as to maximize HIV gene expression while avoiding global $\mathrm{T}$ cell activation. These transcription factors include NF- $\mathrm{B}$ and the HIV transactivator of transcription (Tat) as well as the cyclindependent kinases CDK13 and CDK11 and positive transcription elongation factor $b$ (P-TEFb). Possible therapies involve agents that activate these proteins or release P-TEFb from the inactive 7SK small nuclear ribonucleoprotein (snRNP). These proposed therapies include PKC and MAPK agonists as well as histone deacetylase inhibitors (HDACis) and bromodomain and extraterminal (BET) bromodomain inhibitors (BETis), which act synergistically to reactivate HIV in latently infected cells.
\end{abstract}

\section{Historical perspective}

AIDS first came to public attention in 1981 (1) and represented an acquired immunodeficiency, which invariably led to the demise of the infected individual. Thus, whatever the transmissible agent, the immune system could not eliminate the infection. HIV, which proved to be a retrovirus of the lentivirus family, was identified as the causative agent two years later (2). The provirus was not sequenced until 1985 (3-6). The proviral genome contains genes encoding the viral structural proteins $\mathrm{Gag}$, Pol, and Env, the regulatory proteins HIV transactivator of transcription (Tat) and the regulator of expression of virion proteins (Rev), which are required for HIV replication, and the accessory proteins negative factor ( Nef), viral infectivity factor (Vif), viral protein $\mathrm{U}(\mathrm{Vpu})$, and viral protein $\mathrm{R}$ (Vpr). Vif, Vpu, and Vpr counteract host restriction factors and are not required for HIV replication in permissive cell lines.

Transcription of HIV initiates at the $5^{\prime}$ long terminal repeat (LTR), which acts as a promoter and enhancer. Transcription from the $5^{\prime}$ LTR generates a primary transcript, which is spliced into over 109 mRNAs to produce all viral proteins or is packaged into virions (7). Each LTR contains untranslated 3' (U3), repeat (R), and untranslated $5^{\prime}$ (U5) regions. The $\mathrm{U} 3$ region (453 nt), located at the $5^{\prime}$ end of the LTR, contains cis-acting DNA elements that serve as the binding sites for transcription factors (TFs) including nuclear factor of activated T cells (NFAT), NF- $\kappa$ B, and specificity protein 1 (SP1). This region also contains the TATA box and the initiator element. The transcriptional start site (TSS) is located at the junction between the $\mathrm{U} 3$ and $\mathrm{R}$ regions. The $\mathrm{R}$ region (100 nt), which sits in the middle of the LTR, contains the transactivation response (TAR) element. The U5 region (80 nt) contains the HIV packaging sequences. The $3^{\prime}$ end of the U5 region also contains a lysyl transfer RNA (tRNA) binding site, which serves as the primer for reverse transcription (RT).

Conflict of interest: The authors have declared that no conflict of interest exists. Reference information: / Clin Invest. 2016;126(2):448-454. doi:10.1172/JCI80565.
By 1987, it was clear that the activation of infected cells increased HIV transcription, which followed the translocation of NF- $\mathrm{KB}$ and/ or NFAT into the nucleus (Figure 1 and refs. 8-10). Effects of these TFs are potentiated greatly by Tat, which binds to TAR RNA stem loop rather than to DNA (Figure 1). TAR, which forms after RNA polymerase II (RNAPII) stalls near the TSS, is located at the $5^{\prime}$ end of all HIV transcripts and contains $60 \mathrm{nt}$ (Figure 1). Thus, Tat requires some transcription before it can potentiate HIV gene expression. As such, Tat cannot initiate transcription, but elongates all viral transcripts that contain TAR (11). A new concept in eukaryotic biology was born, one that mirrored the prokaryotic world in which antitermination of transcription regulates, for example, the switch between lysogeny and lysis of bacteriophage $\lambda(11)$.

Because only short transcripts were observed in cells in the absence of Tat and these transcripts were elongated efficiently in the presence of Tat, we proposed that HIV mimicked bacteriophage $\lambda$ and that the virus could lie undetected in cells. Sequential steps of initiation and elongation of transcription also explain the synergy between NF- $\kappa \mathrm{B}$ and Tat. Short, TAR-containing transcripts were also used to detect latently infected cells in patients. Surprisingly, all circulating peripheral blood monocytes (PBMCs) only contained short transcripts at seroconversion (12). However, they could be converted into long transcripts by incubating infected lymphoid cells with allogeneic cells, which activated the latently infected cells via the mixed lymphocyte reaction. In these latently infected cells, HIV replication was induced and fully infectious virus was recovered (12). The reason that circulating lymphocytes did not express any viral proteins lies in the resting nature of these cells, which home to lymphoid organs or sites of inflammation only after activation.

Although these findings clearly demonstrated that latently infected cells could be reactivated, at that time, RT inhibitors were not able to completely suppress HIV replication. A few years later, the addition of protease inhibitors to the antiretroviral therapy (ART) regimen allowed for the suppression of viral RNA below the 


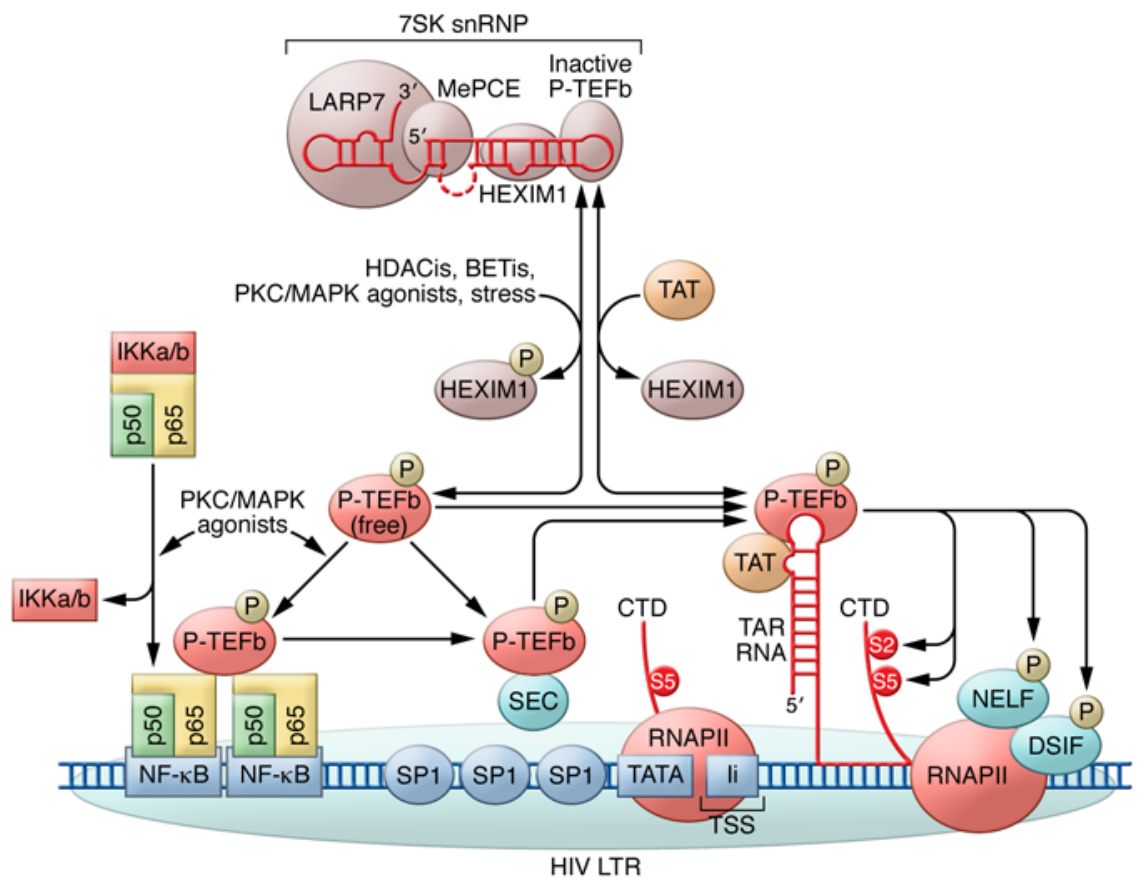

Figure 1. HIV LTR, TFs, and their activation. HIV transcription starts at the TSS, where the initiator element binds. The compact promoter contains a TATA box and 3 SP1 sites. The enhancer contains overlapping NF-KB and nuclear factor of activated T cells (NF-AT) binding sites (NF-AT sites not shown). The promoter recruits RNAPII. During promoter clearance, CTD Ser5 (red circles) is phosphorylated by $\mathrm{CycH} / C D K 7$. P-TEFb is recruited to the HIV LTR via NF- $\mathrm{\kappa B}$ (p50/p65 heterodimer), the super elongation complex (SEC), and Tat. Upon recruitment, P-TEFb phosphorylates Ser2. Tat binds to TAR RNA from positions +1 to +60 in all HIV transcripts. Tat and P-TEFb bind to the $5^{\prime}$ bulge and central loop in TAR, respectively. P-TEFb also phosphorylates Spt5 in DSIF and NELF-E, which releases the arrested RNAPII for elongation. PKC or MAPK agonists promote the translocation of NF- $\mathrm{kB}$ to the nucleus and increase the synthesis of P-TEFb. LRAs as well as stress (apoptosis, UV light, transcriptional blockers) release P-TEFb from the 7SK snRNP, where P-TEFb is inactivated by HEXIM1. Tyr271 in HEXIM1 binds and occludes the ATP pocket in CDK9. Active CDK9 is phosphorylated on Thr186 in the T loop and Ser175. Note the similarity between TAR and the first stem loop in 7SK snRNA, which allows Tat and HEXIM1 to bind to both structures. Tat also competes with HEXIM1 for P-TEFb. Thus, when sufficient amounts of Tat are made, HIV transcription continues despite increased levels of 7SK snRNP.

level of detection. Yet viral DNA persisted, and upon treatment interruption, HIV returned with a vengeance (13-15). Thus, the concepts of proviral latency and HIV reservoir were born and found to be the intractable problem preventing HIV eradication and cure. In subsequent decades, more parameters of the reservoir were defined, i.e., which cells harbor latent proviruses, how many of these are replication competent, the location of the sites of residual replication, etc. Importantly, this knowledge of hidden reservoirs revealed why the immune system and ART cannot eliminate HIV. Only certain HLA haplotypes provide some protection against fulminant disease (16). As to the cure, only heroic measures, such as multiple bone marrow transplants with inhospitable cells, appear to have eliminated the reservoir in a single patient (17).

\section{Basic mechanisms of HIV latency and reservoir}

The latent HIV reservoir is estimated to comprise one in a million resting $\mathrm{CD}^{+} \mathrm{T}$ cells in optimally treated individuals (18), although the total number of these cells could be much higher (19) and is dependent on how soon ART is initiated after infection as well as the duration of the infection (20). In other words, the reservoir expands with HIV replication. In latently infected cells, most provi- ruses are found in actively transcribed genes (21). Several mechanisms could silence HIV gene expression and replication, including (a) deleterious mutations in the viral genome (some of which could be repaired by recombination if more than one virus integrates in the same cell; refs. 22-24), (b) transcriptional interference (25-28), (c) changes in chromatin structure $(29,30)$, (d) epigenetic silencing (such as increased DNA methylation), (e) the presence of negative TFs $(31,32),(\mathrm{f})$ the absence of positive TFs (33-35), and (g) problems with RNA processing and transport (36). These mechanisms could contribute individually and in different combinations to silencing HIV and rendering it invisible to the immune system. Transcriptional interference, heterochromatin and epigenetic alterations, and $\mathrm{TF}$ regulation are discussed in greater detail below.

Transcriptional interference. Replicationcompetent HIV can be silenced in activated as well as in resting cells. The most likely cause of this lack of HIV gene expression is transcriptional interference (Figure 2). In this scenario, HIV is its own worst enemy. Retroviruses have two identical LTRs, one for initiation of transcription ( $5^{\prime}$ LTR), the other for termination of transcription ( $3^{\prime}$ LTR) (37). In the case of HIV, the $5^{\prime}$ LTR initiates transcription 20 times more often than the $3^{\prime}$ LTR, which is occluded by the elongating RNAPII (Figure 2A and ref. 38). Transcriptional interference is partly due to the low-affinity binding between the DNA at the $3^{\prime}$ LTR and SP1, TFIID, and the initiator element $(25,26)$. The elongating RNAPII from the $5^{\prime}$ LTR displaces these TFs from DNA at the $3^{\prime}$ LTR, then continues to the polyA site in the R region and terminates transcription. Notably, when the virus integrates in the same orientation as the host gene, the elongating RNAPII from the host gene terminates at the polyA site in the $5^{\prime}$ LTR, curtailing HIV transcription (25-28). In this case, the $3^{\prime}$ LTR is not occluded and initiates transcription, producing sterile transcripts that contain TAR (Figure 2B). When the virus integrates in the opposite orientation, RNAPII copies HIV antisense transcripts, ignoring both polyA sites in the HIV sense orientation and producing long hybrid mRNA species (Figure $2 \mathrm{C}$ ). If the provirus integrates in introns of host genes, the HIV antisense transcripts are spliced out and rapidly degraded. Nevertheless, HIV antisense transcripts have been detected $(39,40)$, and they could provide an estimate of transcriptional interference in latently infected cells.

The study of transcriptional interference has been facilitated by the use of stably integrated reporter proviruses in Jurkat $\mathrm{T}$ cells, including the J-Lat cell lines (41). If transcriptional interference is robust and persists for the lifetime of the cell, HIV will not be reactivated. However, if the host gene is silenced, then HIV will reactivate. There are several mechanisms by which HIV transcrip- 
A

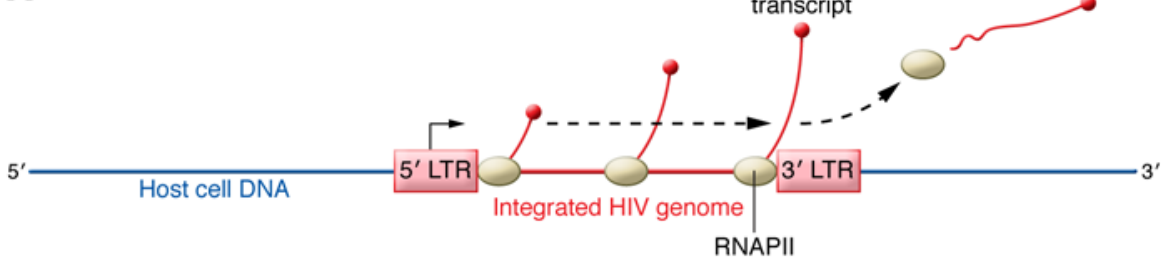

B

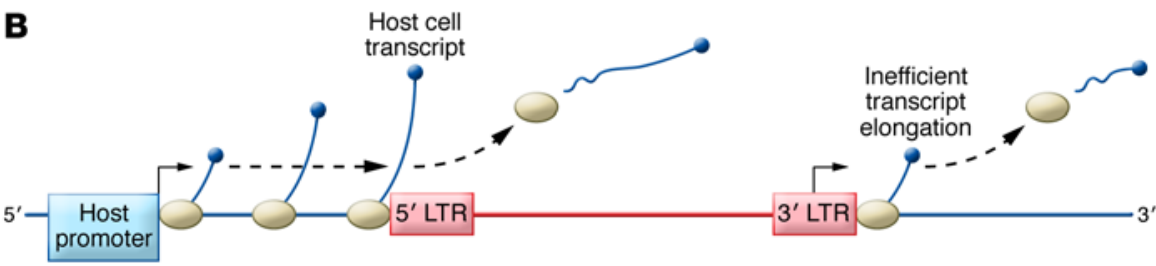

C

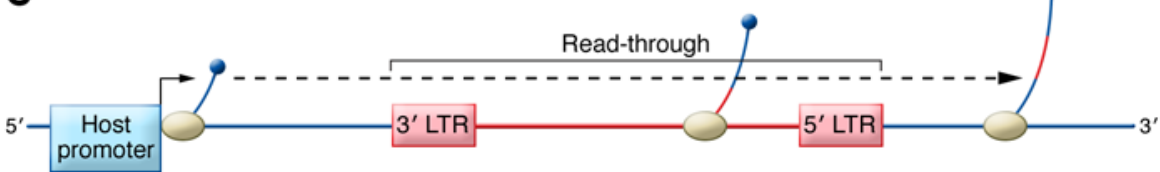

Figure 2. Transcriptional interference. (A) HIV utilizes transcriptional interference for its own replication. The 5' LTR initiates and elongates transcription. The $3^{\prime}$ LTR is occluded because RNAPII displaces TFs from its DNA. Thus, RNAPII terminates past TAR near the polyA site in the $3^{\prime}$ LTR, where CPA of HIV transcripts takes place. However, the same RNAPII transcribes HIV sequences and host cell genes. Thus, when HIV integrates in the sense orientation within a host cell gene, the transcribing RNAPII terminates in the $5^{\prime}$ LTR (B).

The 3' LTR now initiates transcription, which, in the absence of Tat, does not elongate efficiently. When HIV integrates in the antisense orientation within a host gene, RNAPII reads through without stopping (C), and HIV antisense transcripts are released. When integration occurs in introns, HIV RNAs are degraded rapidly. The strength of transcriptional interference depends on rates of transcription of host cell genes. High levels of NF- $\mathrm{KB}$, which bind to DNA tightly, counteract transcriptional interference. Blue and red circles represent 5 ' caps; rising blue and red lines represent mRNA; squiggles represent polyA tails of host cell and HIV mRNAs. tion can be reactivated. In J-Lat 9.2 cells, HIV is integrated in the same orientation as the estrogen-dependent PP5 gene and reactivates upon removal of the hormone (25). Cytokines and the activation state of the infected $\mathrm{T}$ cell also contribute to HIV reactivation

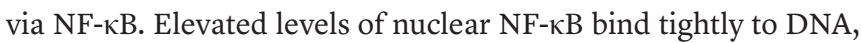
thereby impeding the progress of upstream RNAPII and initiating some transcription from the $5^{\prime}$ LTR. Depending on the degree of transcriptional interference, these processes compete with each other, such that low levels of host gene transcription are overcome more rapidly by cellular activation and vice versa.

Heterochromatin and epigenetic alterations. Integrated proviruses in actively transcribed genes, gene deserts, and Alu-rich repeats can be silenced by heterochromatin. In this scenario, increased levels of DNA methylation, reduced overall cellular gene transcription, increased levels of chromatin silencing complexes, or insufficient positive TFs can potentially contribute to the recruitment of nucleosomes to the viral genome and the HIV LTR (42). Located at the U5 and R regions, nucleosomes inhibit the movement of RNAPII and thus block HIV replication (42). Nevertheless, this silencing can be overcome by activating cells and by increasing Tat synthesis. It is also possible that much of this silencing results from the absence of essential TFs, the levels of which are progressively diminished as activated lymphocytes transition to memory T cells (43-45).

Essential TFs in HIV transcription. In addition to NF- $\mathrm{BB}$ and Tat, other TFs that are expressed at low levels in resting cells are essential for HIV gene expression, including positive transcription elongation factor $\mathrm{b}$ (P-TEFb). P-TEFb was identified by Tat-affinity chromatography (Figure 1 and refs. 33, 34, 46-48) and consists of a large cyclin, CycT1, and a cyclin-dependent kinase, CDK9 $(49,50)$. In the absence of Tat, RNAPII pauses after transcription of TAR. Tat recruits P-TEFb via CycT1 to TAR, allowing CDK9 to phosphorylate the C-terminal domain (CTD) of RNAPII. In addition to $\mathrm{P}-\mathrm{TEFb}$, Tat increases the activity of $\mathrm{CycH} / \mathrm{CDK}$, which also phosphorylates the RNAPII CTD (51). The RNAPII CTD contains 52 YSPTSPS heptapeptide repeats in which all serines, the tyrosine, and the threonine can be phosphorylated by different kinases. These modifications, especially the phosphorylation of serines at position 2 (Ser2P) and 5 (Ser5P), play critical roles in the cotranscriptional processing of nascent mRNA species (52). CycH/CDK7 phosphorylates Ser5, which is required for capping of all viral transcripts (53). More importantly, P-TEFb phosphorylates Ser2, thereby promoting efficient elongation of viral transcription $(50)$.

$\mathrm{P}-\mathrm{TEFb}$ also modulates the activity of factors that impair transcript elongation. P-TEFb phosphorylates the transcription elongation factor SPT5, which is part of the 5,6-dichloro- $1-\beta-D-$ ribofuranosylbenzimidazole (DRB) sensitivity-inducing factor (DSIF) complex and the RD subunit of the negative elongation factor (NELF) complex (NELF-E); these two complexes arrest RNAPII at TAR (54-56). When SPT5 is phosphorylated, DSIF is converted to a positive elongation factor. When NELF-E is phosphorylated, NELF disengages from TAR so that the RNAPII can transition from initiation to elongation (57). Thus, it is not surprising that when levels of P-TEFb are low, HIV is not transcribed. Indeed, this is the case in resting lymphocytes in the periphery of infected individuals as well as in memory $\mathrm{T}$ cells (58). P-TEFb is

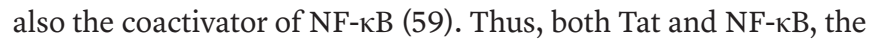
critical regulators of HIV transcription, depend on $\mathrm{P}-\mathrm{TEFb}$.

The regulation of $\mathrm{P}-\mathrm{TEFb}$ itself is of some importance to HIV transcription. As mentioned above, levels of CycT1 are exceedingly low in resting cells. Regulation of CycT1 expression is posttranscriptional and depends on specific miRNAs that inhibit the translation of CYCT1 mRNA by binding to its $3^{\prime}$ UTR (45). Cell activation relieves this block, and levels of $\mathrm{P}-\mathrm{TEFb}$ rise rapidly (45, 60); however, most P-TEFb is sequestered and inactivated in the 7SK small nuclear ribonucleoprotein (7SK snRNP) (Figure 1 and refs. 61, 62). Methylphosphate capping enzyme (MePCE) and Larelated protein 7 (LARP7) bind and protect the $5^{\prime}$ and $3^{\prime}$ ends of 


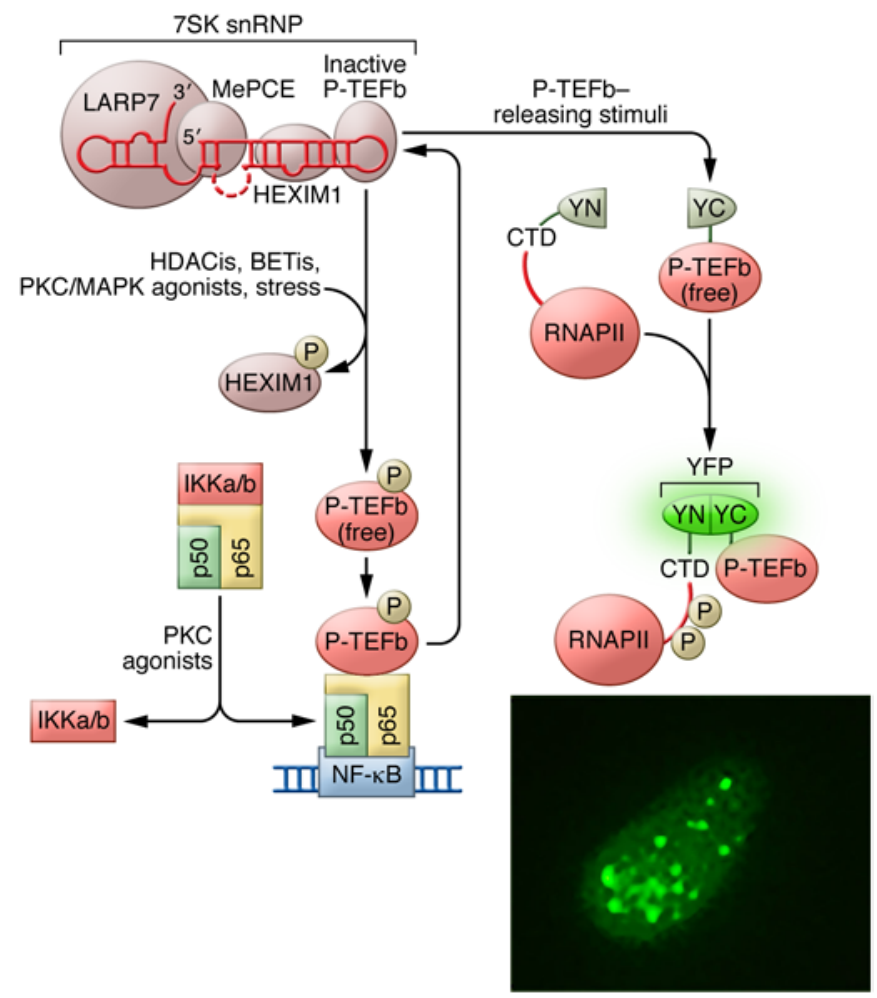

7SK small nuclear RNA 7SK (snRNA) from degradation. The $5^{\prime}$ RNA stem loop of 7SK snRNP resembles TAR $(57,63)$ and is bound by cellular hexamethylene bis-acetamide-inducible proteins (HEXIM1/2), which undergo a conformational change upon RNA binding. RNA-associated HEXIMs then bind and inhibit P-TEFb in the 7SK snRNP. Notably, the rapid release of $\mathrm{P}-\mathrm{TEFb}$ from the 7SK snRNP also increases the synthesis of HEXIM1, which reassembles the 7SK snRNP and sequesters free P-TEFb (64). The ratio of free and bound $\mathrm{P}-\mathrm{TEFb}$ (the $\mathrm{P}-\mathrm{TEFb}$ equilibrium) then determines the state of cell growth, proliferation, and differentiation (65). Although cell activation increases levels of P-TEFb sufficiently to support HIV transcription (58), this effect is augmented when $\mathrm{P}-\mathrm{TEFb}$ is released rapidly from the 7SK snRNP. This release follows cell stress, which can come in the form of apoptosis, radiation, UV light, and compounds that block transcription (actinomycin D, DRB, or flavopiridol) as well as chemicals that cause changes in chromatin (histone deacetylase inhibitors [HDACis] and bromodomain and extraterminal [BET] bromodomain inhibitors [BETis]) and affect DNA methylation (5-azacytidine) (43, 61, 62). Importantly, these latency reversing agents (LRAs) only work when P-TEFb is abundant and present in the 7SK snRNP (43).

$\mathrm{CycH} / \mathrm{CDK} 7$ and P-TEFb are not sufficient for optimal cotranscriptional processing of viral transcripts, which is necessary for HIV gene expression; additional transcriptional CDKs, including $\mathrm{CycK} / \mathrm{CDK} 13$ and $\mathrm{CycL} / \mathrm{CDK} 11$, are required (66-68). Both of these complexes increase levels of Ser2P at HIV coding and 3' end sequences $(69,70)$. Levels of CDK13 affect HIV splicing, and high and low levels of CDK13 increase and decrease splicing of HIV mRNA, respectively (66). Only the unspliced transcripts lead to the production of infectious viral particles. In contrast, CDK11 does not significantly affect HIV mRNA splicing (68); instead, it affects
Figure 3. Combinatorial activation of TFs for HIV reactivation from latency. Mechanisms of action of PKC agonists and LRAs for increased HIV gene expression as well as the V-PAC assay are shown. PKC agonists activate NF- $\kappa B$ and increase levels of transcriptional CDKs, P-TEFb, CycK/ CDK13, and CyCL/CDK11. LRAs release P-TEFb from the 7SK snRNP. This rapid release is followed by the reassembly of the 7SK snRNP, which returns activated cells to their resting states. Tat interrupts this P-TEFb cycle (see Figure 1). V-PAC measures the release of P-TEFb from the 7SK snRNP in living cells; thus, it measures the strength and kinetics of LRA effects. In this BiFC, the two halves of YFP are linked to the CTD of RNAPII and P-TEFb. When P-TEFb is released and phosphorylates the CTD, cells turn green, especially in nuclear speckles, which are sites of active transcription (inset).

the cleavage and polyadenylation (CPA) of all HIV viral transcripts. By increasing levels of Ser2P near the HIV 3' end, CDK11 helps to recruit CPA factors to RNAPII, which directs the appropriate CPA of all viral transcripts. Longer polyA tails lead to greater stability of these mRNA species and their improved export and translation in the cytoplasm. Importantly, CDK11 levels are also very low in resting cells and increase following T cell activation (67).

All of these RNAPII CTD kinases must work in harmony with each other, and their imbalances can have deleterious effects. For example, when LARP7 or 7SK snRNA are reduced in stem cells, levels of free P-TEFb rise and overall transcription is increased (71). However, RNAPII does not terminate transcription efficiently, allowing for read-through of megabases of DNA, which results in extremely long transcripts that contain many genes (72). Protein products of these transcripts are not expressed, as only proper RNA processing ensures the stability, transport, and translation of genes. Thus, levels of other necessary CTD kinases are not sufficiently high for appropriate mRNA splicing and CPA in these cells. This scenario may also occur if P-TEFb alone is increased by specific interventions. In addition, because the stalled RNAPII requires $\mathrm{P}-\mathrm{TEFb}$ for elongation, levels of $\mathrm{P}-\mathrm{TEFb}$ must rise before those for CDK11 or CDK13. Thus, at least in the initial phases of HIV reactivation, most viral RNA species could represent readthrough transcripts from host cell genes.

To ensure proper transcription and cotranscriptional processing of HIV, CDK expression must be coordinately upregulated. T cell activation, which occurs via antigen stimulation of the $\mathrm{T}$ cell receptor and engagement of costimulatory receptors, accomplishes this task efficiently. CDK expression is required to overcome T cell quiescence and reactivate HIV in these previously resting cells (58); however, such activation also results in the release of delete- 
rious lymphokines and cytokines, resulting in cytokine storm with concomitant hypotension and shock. Thus, more gentle methods of $\mathrm{T}$ cell activation are required. Indeed, specific PKC agonists can also increase levels of CTD kinases. These compounds work synergistically with LRAs that release P-TEFb from the 7SK snRNP (Figure 3 and refs. 73, 74). Release of P-TEFb by LRAs induces the synthesis of HEXIM1, which reassembles the 7SK snRNP and inhibits free P-TEFb (64). Because lymphokine and cytokine genes require active $\mathrm{P}-\mathrm{TEFb}$ for their expression, HDACis and BETis also ensure that there is no cytokine storm (75).

\section{Diagnostic and therapeutic implications}

Given these mechanistic details about HIV and eukaryotic transcription, it would be useful to translate them to clinical strategies for the cure of AIDS. First, there are diagnostic considerations. Stalled transcription at $5^{\prime}$ or $3^{\prime}$ LTRs and the release of short transcripts that contain TAR can serve as surrogate marker for measurements of the viral reservoir. For example, one can detect these short RNA species in the blood, in PBMCs, and in exosomes that are released from infected cells $(12,76,77)$. In one study, TAR exosomes were even more abundant in elite controllers, infected individuals with undetectable levels of viral RNA species in their plasma (77). Short HIV transcripts also reveal the presence of latently infected circulating cells (12). Comparing transcript ratios in tissues and blood could yield an approximation of the viral reservoir in the infected host. Importantly, once those levels are established, it should be possible to monitor these ratios and assess the clinical effectiveness of any HIV anti-latency therapy (HALT).

We also need to engineer useful tools for developing effective HALT. Cell line models of latency, such as J-Lat cells, have traditionally been used to screen for potential compounds (78). Now, primary cells must also be examined. Activation and translocation of NF- $\mathrm{BB}$ can be followed by reporter gene and immunofluorescent assays (IFAs) in living cells. Recently, we developed an assay for P-TEFb equilibrium in which we follow the recruitment of P-TEFb to the CTD of RNAPII. We call this assay visualization of P-TEFb activation in living cells (V-PAC) (Figure 3 and ref. 79). Using bimolecular fluorescence complementation (BiFC) (80), we follow the speed and extent of P-TEFb release from the 7SK snRNP in real time. The V-PAC assay can also be adapted for the screening of compounds that could be used for the "shock and kill" strategy of HIV elimination. Importantly, all compounds that score positive in the V-PAC assay also contribute to HIV reactivation in primary cells, including models of proviral latency or resting cells from optimally treated, HIV-infected individuals. As mentioned above, HEXIM1 is most sensitive to levels of free $\mathrm{P}-\mathrm{TEFb}$ and is synthesized rapidly upon the disruption of the 7SK snRNP. Thus, V-PAC can be complimented by a reporter assay in which the promoter of HEXIM1 is placed upstream of the firefly luciferase gene. Additionally, IFAs, Western blotting, and flow cytometry with antibodies that detect phosphorylation of S175 have been used to detect the activation of P-TEFb (81). Thus far, there are no assays to detect levels and activation of CDK11 or CDK13. Nevertheless, similar assays can be developed to follow their levels and activities in cells. Although specific targets have to be introduced into cells in all these reporter systems, these approaches are now possible with primary cells, including resting $\mathrm{T}$ cells. By using liposomes loaded with Vpx to eliminate the HIV restriction factor SamHD1, reporter lentiviruses can be introduced into resting primary $\mathrm{CD} 4^{+}$lymphocytes (82).

Measurements of expression levels and activities of critical TFs will suggest combinations of compounds that could be used for HIV reactivation from latency (Figure 3). Because amounts of transcriptional CDKs are low in resting cells, they must be increased for HIV gene expression (58). PKC agonists, including phorbol esters, prostratin, bryostatin, and ingenol, can accomplish this task $(74,83-88)$. These compounds have appreciable toxicity, and most of them cannot be administered orally. An exception is ingenol B, which was modified from other ingenols obtained from the euphorbia plant. Oral administration of ingenol B to optimally treated, infected rhesus macaques activated cells and increased levels of SIV in the periphery $(86,87,89,90)$.

Combinations of PKC or MAPK agonists and LRAs will be needed to mitigate toxic effects and lower effective doses $(73,74$, 91). Such LRAs include HDACis, BETis, and 5' azacytidine, among others. Indeed, vorinostat, romidepsin, panobinostat, and JQ1 act synergistically with PKC agonists to reactivate HIV transcription (43, 91-95). Whereas PKC agonists activate NF-кB and increase overall levels of $\mathrm{P}-\mathrm{TEFb}$ and $\mathrm{CycL} / \mathrm{CDK} 11, \mathrm{LRAs}$ primarily affect the P-TEFb equilibrium. By releasing free P-TEFb, LRAs also induce the synthesis of HEXIM1, which results in the rapid reassembly of the 7SK snRNP. Thus, LRAs limit the concomitant production of cytokines induced by PKC agonists. Most importantly, lower concentrations of PKC agonists and LRAs can be used in combination to achieve optimal HIV reactivation $(73,91)$. Time of administration might also be an important therapeutic component, as PKC agonists and LRAs act through different mechanisms. By first increasing cellular levels of P-TEFb, PKC agonists could facilitate the ability of LRAs to release $\mathrm{P}-\mathrm{TEFb}$ from $7 \mathrm{SK}$ snRNP. This one-two punch should be explored further in primary cells, patient cells ex vivo, and infected rhesus macaques. It is possible that sustained, low levels of ART and HALT will diminish the reservoir of HIV and lead to a functional cure of the infection, helping to convert most infected individuals to elite controllers.

\section{Summary}

Much has been learned about the replicative cycle of HIV. A critical component is its ability to establish proviral latency in infected cells, which was revealed by studies in transformed cell lines, primary cells, and infected individuals. Containing and/or removing this viral reservoir is a daunting problem for eukaryotic biology and clinical science. It is hoped that sustained reactivation of HIV in the presence of optimal ART and adequate immune responses will prevent de novo infections and remove HIV, albeit slowly, from the infected host.

\section{Acknowledgments}

We thank members of the Peterlin laboratory. This work was supported by NIH grants U19 AI096113 (CARE Center Grant, David Margolis, PI), P50 GM082250 (HARC Center Grant, Alan Frankel, and Nevan Krogan, co-PIs), and AI1049104 (to B.M. Peterlin). K. Fujinaga was supported by the California HIV/AIDS Research Program grant ID-13-SF-558.

Address correspondence to: B. Matija Peterlin, Box 0703, Rm U432 UCSF, 533 Parnassus Ave., San Francisco, California 941430703, USA. Phone: 415.502.1905; E-mail: matija.peterlin@ucsf.edu. 
1. Centers for Disease Control (CDC). Pneumocystis pneumonia - Los Angeles. MMWR Morb Mortal Wkly Rep. 1981;30(21):250-252.

2. Barre-Sinoussi F, et al. Isolation of a T-lymphotropic retrovirus from a patient at risk for acquired immune deficiency syndrome (AIDS). Science. 1983;220(4599):868-871.

3. Alizon M, et al. Molecular cloning of lymphadenopathy-associated virus. Nature. 1984;312(5996):757-760.

4. Sanchez-Pescador R, et al. Nucleotide sequence and expression of an AIDS-associated retrovirus (ARV-2). Science. 1985;227(4686):484-492.

5. Arya SK, Guo C, Josephs SF, Wong-Staal F. Transactivator gene of human T-lymphotropic virus type III (HTLV-III). Science. 1985;229(4708):69-73.

6. Sodroski J, et al. Trans-acting transcriptional regulation of human T-cell leukemia virus type III long terminal repeat. Science. 1985;227(4683):171-173.

7. Ocwieja KE, et al. Dynamic regulation of HIV-1 mRNA populations analyzed by single-molecule enrichment and long-read sequencing. Nucleic Acids Res. 2012;40(20):10345-10355.

8. Nabel G, Baltimore D. An inducible transcription factor activates expression of human immunodeficiency virus in T cells. Nature. 1987;326(6114):711-713.

9. Tong-Starksen SE, Luciw PA, Peterlin BM. Human immunodeficiency virus long terminal repeat responds to T-cell activation signals. Proc Natl Acad Sci U S A . 1987;84(19):6845-6849.

10. Kinoshita S, Su L, Amano M, Timmerman LA, Kaneshima H, Nolan GP. The T cell activation factor NF-ATc positively regulates HIV-1 replication and gene expression in T cells. Immunity. 1997;6(3):235-244.

11. Kao SY, Calman AF, Luciw PA, Peterlin BM. Anti-termination of transcription within the long terminal repeat of HIV-1 by tat gene product. Nature. 1987;330(6147):489-493.

12. Adams M, et al. Cellular latency in human immunodeficiency virus-infected individuals with high CD4 levels can be detected by the presence of promoter-proximal transcripts. Proc Natl Acad Sci US A. 1994;91(9):3862-3866.

13. Chun TW, et al. Presence of an inducible HIV-1 latent reservoir during highly active antiretroviral therapy. Proc Natl Acad Sci U S A. 1997;94(24):13193-13197.

14. Finzi D, et al. Identification of a reservoir for HIV-1 in patients on highly active antiretroviral therapy. Science. 1997;278(5341):1295-1300.

15. Wong JK, et al. Recovery of replication-competent HIV despite prolonged suppression of plasma viremia. Science. 1997;278(5341):1291-1295.

16. Altfeld M, et al. HLA alleles associated with delayed progression to AIDS contribute strongly to the initial CD8(+) T cell response against HIV-1. PLoS Med. 2006;3(10):e403.

17. Hutter $\mathrm{G}$, et al. Long-term control of HIV by CCR5 $\Delta 32 / \Delta 32$ stem-cell transplantation. $N$ Engl JMed. 2009;360(7):692-698.

18. Eriksson S, et al. Comparative analysis of measures of viral reservoirs in HIV-1 eradication studies. PLoS Pathog. 2013;9(2):e1003174.

19. Ho YC, et al. Replication-competent noninduced proviruses in the latent reservoir increase barrier to HIV-1 cure. Cell. 2013;155(3):540-551.

20. Archin NM, et al. HIV-1 expression within resting $\mathrm{CD}^{+} \mathrm{T}$ cells after multiple doses of vorinostat. J Infect Dis. 2014;210(5):728-735.

21. Han $Y$, et al. Resting $C D 4^{+} \mathrm{T}$ cells from human immunodeficiency virus type 1 (HIV-1)-infected individuals carry integrated HIV-1 genomes within actively transcribed host genes. JVirol. 2004;78(12):6122-6133.

22. Jung $A$, et al. Recombination: Multiply infected spleen cells in HIV patients. Nature. 2002;418(6894):144.

23. Emiliani S, Fischle W, Ott M, Van Lint C, Amella $\mathrm{CA}$, Verdin E. Mutations in the tat gene are responsible for human immunodeficiency virus type 1 postintegration latency in the U1 cell line. JVirol.1998;72(2):1666-1670.

24. Emiliani S, et al. A point mutation in the HIV-1 Tat responsive element is associated with postintegration latency. Proc Natl Acad Sci U S A. 1996;93(13):6377-6381

25. Lenasi T, Contreras X, Peterlin BM. Transcriptional interference antagonizes proviral gene expression to promote HIV latency. Cell Host Microbe. 2008;4(2):123-133.

26. Greger IH, Demarchi F, Giacca M, Proudfoot NJ. Transcriptional interference perturbs the binding of Sp1 to the HIV-1 promoter. Nucleic Acids Res. 1998;26(5):1294-1301.

27. Ashe MP, Griffin P, James W, Proudfoot NJ. Poly(A) site selection in the HIV-1 provirus: inhibition of promoter-proximal polyadenylation by the downstream major splice donor site. Genes Dev. 1995;9(23):3008-3025.

28. Han Y, et al. Orientation-dependent regulation of integrated HIV-1 expression by host gene transcriptional readthrough. Cell Host Microbe. 2008;4(2):134-146.

29. Kauder SE, Bosque A, Lindqvist A, Planelles $\mathrm{V}$, Verdin E. Epigenetic regulation of HIV-1 latency by cytosine methylation. PLoS Pathog. 2009;5(6):e1000495.

30. Mbonye U, Karn J. Transcriptional control of HIV latency: cellular signaling pathways, epigenetics, happenstance and the hope for a cure. Virology. 2014;454-455:328-339.

31. Zhang Z, Klatt A, Gilmour DS, Henderson AJ. Negative elongation factor NELF represses human immunodeficiency virus transcription by pausing the RNA polymerase II complex. J Biol Chem. 2007;282(23):16981-16988.

32. Jadlowsky JK, et al. Negative elongation factor is required for the maintenance of proviral latency but does not induce promoter-proximal pausing of RNA polymerase II on the HIV long terminal repeat. Mol Cell Biol. 2014;34(11):1911-1928.

33. Rice AP, Herrmann CH. Regulation of TAK/ $\mathrm{P}-\mathrm{TEFb}$ in CD4+ $\mathrm{T}$ lymphocytes and macrophages. Curr HIV Res. 2003;1(4):395-404.

34. Yang $X$, et al. TAK, an HIV Tat-associated kinase, is a member of the cyclin-dependent family of protein kinases and is induced by activation of peripheral blood lymphocytes and differentiation of promonocytic cell lines. Proc Natl Acad Sci U S A. 1997;94(23):12331-12336.

35. Williams SA, Greene WC. Host factors regulating post-integration latency of HIV. Trends Microbiol. 2005;13(4):137-139.
36. Lassen KG, Ramyar KX, Bailey JR, Zhou Y, Siliciano RF. Nuclear retention of multiply spliced HIV-1 RNA in resting $\mathrm{CD}^{+} \mathrm{T}$ cells. PLoS Pathog. 2006;2(7):e68.

37. Cullen BR, Lomedico PT, Ju G. Transcriptional interference in avian retroviruses - implications for the promoter insertion model of leukaemogenesis. Nature. 1984;307(5948):241-245.

38. Klaver B, Berkhout B. Comparison of $5^{\prime}$ and $3^{\prime}$ long terminal repeat promoter function in human immunodeficiency virus. J Virol. 1994;68(6):3830-3840.

39. Landry S, et al. Detection, characterization and regulation of antisense transcripts in HIV-1. Retrovirology. 2007;4:71.

40. Ludwig LB, et al. Human Immunodeficiency Virus-Type 1 LTR DNA contains an intrinsic gene producing antisense RNA and protein products. Retrovirology. 2006;3:80.

41. Jordan A, Bisgrove D, Verdin E. HIV reproducibly establishes a latent infection after acute infection of T cells in vitro. EMBO J. 2003;22(8):1868-1877.

42. Verdin E, Paras P Jr, Van Lint C. Chromatin disruption in the promoter of human immunodeficiency virus type 1 during transcriptional activation. EMBO J. 1993;12(8):3249-3259.

43. Bartholomeeusen K, Fujinaga K, Xiang Y, Peterlin BM. Histone deacetylase inhibitors (HDACis) that release the positive transcription elongation factor $\mathrm{b}(\mathrm{P}-\mathrm{TEFb})$ from its inhibitory complex also activate HIV transcription.J Biol Chem. 2013;288(20):14400-14407.

44. Budhiraja S, Rice AP. Reactivation of latent HIV: do all roads go through P-TEFb? Future Virol. 2013;8(7):10.2217/fvl.13.52

45. Chiang K, Rice AP. MicroRNA-mediated restriction of HIV- 1 in resting $\mathrm{CD} 4^{+} \mathrm{T}$ cells and monocytes. Viruses. 2012;4(9):1390-1409.

46. Marshall NF, Price DH. Purification of P-TEFb, a transcription factor required for the transition into productive elongation. J Biol Chem. 1995;270(21):12335-12338.

47. Zhu Y, et al. Transcription elongation factor $\mathrm{P}-\mathrm{TEFb}$ is required for HIV-1 tat transactivation in vitro. Genes Dev. 1997;11(20):2622-2632.

48. Mancebo HS, et al. P-TEFb kinase is required for HIV Tat transcriptional activation in vivo and in vitro. Genes Dev. 1997;11(20):2633-2644.

49. Garber ME, et al. The interaction between HIV-1 Tat and human cyclin T1 requires zinc and a critical cysteine residue that is not conserved in the murine CycT1 protein. Genes Dev. 1998;12(22):3512-3527.

50. Wei P, Garber ME, Fang SM, Fischer WH, Jones KA. A novel CDK9-associated C-type cyclin interacts directly with HIV-1 Tat and mediates its high-affinity, loop-specific binding to TAR RNA. Cell.1998;92(4):451-462.

51. Cujec TP, et al. The HIV transactivator TAT binds to the CDK-activating kinase and activates the phosphorylation of the carboxy-terminal domain of RNA polymerase II. Genes Dev. 1997;11(20):2645-2657.

52. Heidemann M, Hintermair C, Voss K, Eick D. Dynamic phosphorylation patterns of RNA polymerase II CTD during transcription. Biochim Biophys Acta. 2013;1829(1):55-62.

53. Zhou M, Deng L, Kashanchi F, Brady JN, Shatkin 
AJ, Kumar A. The Tat/TAR-dependent phosphorylation of RNA polymerase II C-terminal domain stimulates cotranscriptional capping of HIV-1 mRNA. Proc Natl Acad Sci U S A. 2003;100(22):12666-12671.

54. Yamaguchi Y, Inukai N, Narita T, Wada T, Handa $\mathrm{H}$. Evidence that negative elongation factor represses transcription elongation through binding to a DRB sensitivity-inducing factor/RNA polymerase II complex and RNA. Mol Cell Biol. 2002;22(9):2918-2927.

55. Yamaguchi Y, Shibata H, Handa H. Transcription elongation factors DSIF and NELF: promoterproximal pausing and beyond. Biochim Biophys Acta. 2013;1829(1):98-104.

56. Renner DB, Yamaguchi Y, Wada T, Handa H, Price DH. A highly purified RNA polymerase II elongation control system. J Biol Chem. 2001;276(45):42601-42609.

57. Fujinaga K, Irwin D, Huang Y, Taube R, Kurosu T, Peterlin BM. Dynamics of human immunodeficiency virus transcription: $\mathrm{P}-\mathrm{TEFb}$ phosphorylates $\mathrm{RD}$ and dissociates negative effectors from the transactivation response element. Mol Cell Biol. 2004;24(2):787-795.

58. Rice AP. P-TEFb as a target to reactivate latent HIV: two Brds are now in hand. Cell Cycle. 2013;12(3):392-393.

59. Barboric M, Nissen RM, Kanazawa S, JabraneFerrat N, Peterlin BM. NF-kappaB binds P-TEFb to stimulate transcriptional elongation by RNA polymerase II. Mol Cell. 2001;8(2):327-337.

60. Chiang K, Sung TL, Rice AP. Regulation of cyclin T1 and HIV-1 Replication by microRNAs in resting $\mathrm{CD}^{+}{ }^{+} \mathrm{T}$ lymphocytes. JVirol. 2012;86(6):3244-3252.

61. Zhou Q, Yik JH. The Yin and Yang of P-TEFb regulation: implications for human immunodeficiency virus gene expression and global control of cell growth and differentiation. Microbiol Mol Biol Rev. 2006;70(3):646-659.

62. Peterlin BM, Brogie JE, Price DH. 7SK snRNA: a noncoding RNA that plays a major role in regulating eukaryotic transcription. Wiley Interdiscip Rev RNA. 2012;3(1):92-103.

63. Muniz L, Egloff S, Ughy B, Jady BE, Kiss T. Controlling cellular P-TEFb activity by the HIV-1 transcriptional transactivator Tat. PLoS Pathog. 2010;6(10):e1001152.

64. Liu P, et al. Release of positive transcription elongation factor $b(\mathrm{P}-\mathrm{TEFb})$ from 7SK small nuclear ribonucleoprotein (snRNP) activates hexamethylene bisacetamide-inducible protein (HEXIM1) transcription. J Biol Chem. 2014;289(14):9918-9925.

65. He N, Pezda AC, Zhou Q. Modulation of a $\mathrm{P}-\mathrm{TEFb}$ functional equilibrium for the global control of cell growth and differentiation. $\mathrm{Mol}$ Cell Biol. 2006;26(19):7068-7076.

66. Berro R, et al. CDK13, a new potential human immunodeficiency virus type 1 inhibitory factor regulating viral mRNA splicing. J Virol. 2008;82(14):7155-7166.
67. Yu W, Ramakrishnan R, Wang Y, Chiang K, Sung TL, Rice AP. Cyclin T1-dependent genes in activated $\mathrm{CD} 4 \mathrm{~T}$ and macrophage cell lines appear enriched in HIV-1 co-factors. PLoS One. 2008;3(9):e3146.

68. Pak V, Eifler TT, Jäger S, Krogan NJ, Fujinaga K, Peterlin BM. CDK11 in TREX/THOC regulates HJIV mRNA 3' end processing. Cell Host Microbe. 2015;18(5):560-570.

69. Liang K, et al. Characterization of human cyclindependent kinase 12 (CDK12) and CDK13 complexes in C-terminal domain phosphorylation, gene transcription, and RNA processing. Mol Cell Biol. 2015;35(6):928-938.

70. Trembley JH, Hu D, Slaughter CA, Lahti JM, Kidd VJ. Casein kinase 2 interacts with cyclindependent kinase 11 (CDK11) in vivo and phosphorylates both the RNA polymerase II carboxylterminal domain and CDK11 in vitro. J Biol Chem. 2003;278(4):2265-2270.

71. Dai Q, et al. Primordial dwarfism gene maintains Lin28 expression to safeguard embryonic stem cells from premature differentiation. Cell Rep. 2014;7(3):735-746.

72. Castelo-Branco G, et al. The non-coding snRNA 7SK controls transcriptional termination, poising, and bidirectionality in embryonic stem cells. Genome Biol. 2013;14(9):R98.

73. Laird GM, et al. Ex vivo analysis identifies effective HIV-1 latency-reversing drug combinations. JClin Invest. 2015;125(5):1901-1912.

74. Bullen CK, Laird GM, Durand CM, Siliciano JD, Siliciano RF. New ex vivo approaches distinguish effective and ineffective single agents for reversing HIV-1 latency in vivo. Nat Med. 2014;20(4):425-429.

75. Li N, et al. HDAC inhibitor reduces cytokine storm and facilitates induction of chimerism that reverses lupus in anti-CD3 conditioning regimen. Proc Natl Acad Sci U S A. 2008;105(12):4796-4801.

76. Lassen KG, Bailey JR, Siliciano RF. Analysis of human immunodeficiency virus type 1 transcriptional elongation in resting $\mathrm{CD}^{+}{ }^{+} \mathrm{T}$ cells in vivo. JVirol. 2004;78(17):9105-9114.

77. Narayanan A, et al. Exosomes derived from HIV-1-infected cells contain trans-activation response element RNA. J Biol Chem. 2013;288(27):20014-20033.

78. Gallastegui E, et al. Combination of biological screening in a cellular model of viral latency and virtual screening identifies novel compounds that reactivate HIV-1. J Virol. 2012;86(7):3795-3808.

79. Fujinaga K, Luo Z, Schaufele F, Peterlin BM. Visualization of positive transcription elongation factor b (P-TEFb) activation in living cells. J Biol Chem. 2015;290(3):1829-1836.

80. Kerppola TK. Bimolecular fluorescence complementation (BiFC) analysis as a probe of protein interactions in living cells. Annu Rev Biophys. 2008;37:465-487.

81. Mbonye UR, et al. Phosphorylation of CDK9 at Ser175 enhances HIV transcription and is a mark- er of activated P-TEFb in CD4(+) T lymphocytes. PLoS Pathog. 2013;9(5):e1003338.

82. Geng X, Doitsh G, Yang Z, Galloway NL, Greene WC. Efficient delivery of lentiviral vectors into resting human CD4 T cells. Gene Ther. 2014;21(4):444-449.

83. Beans EJ, et al. Highly potent, synthetically accessible prostratin analogs induce latent HIV expression in vitro and ex vivo. Proc Natl Acad Sci U S A. 2013;110(29):11698-11703.

84. Korin YD, Brooks DG, Brown S, Korotzer A, Zack JA. Effects of prostratin on T-cell activation and human immunodeficiency virus latency. J Virol. 2002;76(16):8118-8123.

85. Perez M, et al. Bryostatin-1 synergizes with histone deacetylase inhibitors to reactivate HIV-1 from latency. Curr HIV Res. 2010;8(6):418-429.

86. Jiang G, et al. Reactivation of HIV latency by a newly modified Ingenol derivative via protein kinase $\mathrm{C} \Delta-\mathrm{NF}-\mathrm{kB}$ signaling. AIDS. 2014;28(11):1555-1566

87. Pandelo Jose D, et al. Reactivation of latent HIV-1 by new semi-synthetic ingenol esters. Virology. 2014;462-463:328-339.

88. Hori T, et al. Procyanidin trimer $\mathrm{C} 1$ derived from Theobroma cacao reactivates latent human immunodeficiency virus type 1 provirus. Biochem Biophys Res Commun. 2015;459(2):288-293.

89. Abreu CM, et al. Dual role of novel ingenol derivatives from Euphorbia tirucalli in HIV replication: inhibition of de novo infection and activation of viral LTR. PLoS One. 2014;9(5):e97257.

90. Gama L, et al. Latency reversing agents activate latent reservoirs in the brain of SIV-infected macaques. Presented at: Conference on Retroviruses and Opportunistic Infections; February 23-26, 2015; Seattle, Washington, USA. Abstract 416.

91. Darcis G, et al. An in-depth comparison of latencyreversing agent combinations in various in vitro and ex vivo HIV-1 latency models identified Bryostatin-1+JQ1 and Ingenol-B+JQ1 to Potently reactivate viral gene expression. PLoS Pathog. 2015;11(7):e1005063.

92. Bartholomeeusen K, Xiang Y, Fujinaga K, Peterlin BM. Bromodomain and extra-terminal (BET) bromodomain inhibition activate transcription via transient release of positive transcription elongation factor $\mathrm{b}(\mathrm{P}-\mathrm{TEFb})$ from 7SK small nuclear ribonucleoprotein. J Biol Chem. 2012;287(43):36609-36616.

93. Contreras X, et al. Suberoylanilide hydroxamic acid reactivates HIV from latently infected cells. J Biol Chem. 2009;284(11):6782-6789.

94. Rasmussen TA, et al. Comparison of HDAC inhibitors in clinical development: effect on HIV production in latently infected cells and T-cell activation. Hum Vaccin Immunother. 2013;9(5):993-1001.

95. Wei DG, et al. Histone deacetylase inhibitor romidepsin induces HIV expression in CD4 T cells from patients on suppressive antiretroviral therapy at concentrations achieved by clinical dosing. PLoS Pathog. 2014;10(4):e1004071. 